\title{
On the Role of Short and Strong Hydrogen Bonds on the Mechanism of Action of a Model Chymotrypsine Active Site
}

\author{
German Miño*,† and Renato Contreras ${ }^{\ddagger}$ \\ Department of Chemistry, Faculty of Ecology and Natural Resources, Universidad Andrés Bello, Avenida \\ República 257, Santiago, Chile and Departamento de Química, Facultad de Ciencias, Universidad de Chile, \\ Casilla 653, Santiago, Chile
}

Received: February 17, 2009

The electronic properties of a chemical model that mimics the His-57 $\cdots$ Asp-102 catalytic residues of $\alpha$ chymotrypsine during the transition from normal hydrogen bond to short and strong hydrogen-bond regimes are presented. The results suggest that upon a global external stimulus induced by compression, the system response is the transfer of the nucleophilic reactivity from the model Asp-102 moiety toward the model His-57 fragment in the hydrogen-bonded complex. In this way, the catalytic effect may be consistently explained on the basis of a pair site reactivity model framed on the second-order static density response function.

\section{Introduction}

Low-barrier hydrogen bonds (lbhb), also described as short and strong hydrogen bonds (sshb), have been proposed to participate in many chemical and biochemical processes. ${ }^{1-8}$ The acronym sshb comes from the fact that this kind of structure has unusual donor-acceptor hydrogen-bond (HB) distances, ranging from 2.40 to $2.65 \AA$, and formation enthalpies within the range from 12 to $24 \mathrm{kcal} / \mathrm{mol}$. These sshb markedly differ from normal or weak HB, with distances from 2.65 to $3.0 \AA$ and formation enthalpies ranging from 2.5 to $12 \mathrm{kcal} / \mathrm{mol} .{ }^{1}$ Under normal $\mathrm{HB}$ conditions, the potential that confines the proton shows a double-well potential separated by a barrier that is higher than the zero-point energy of the $\mathrm{H}$ atom. ${ }^{1}$ When the $\mathrm{HB}$ distance is shortened, the barriers are dramatically diminished below the $\mathrm{H}$ zero-point vibrational energy and the lbhb denomination applies. ${ }^{2}$

An extensive body of research has correlated the appearance of sshb with some biocatalysts that form these unusually strong H-bonded structures. ${ }^{2-6}$ In the particular case of the bovine protease $\alpha$-chymotrypsine, composed by aspartate-102 (Asp-102), histidine57 (His-57), and serine-195 (Ser-195) (see Scheme 1), Frey et al. ${ }^{6-9}$ proposed a mechanism for the chemical activation promoted by this enzyme that involves the formation of an sshb.

In this mechanism, the substrate binding induces a steric compression between His-57 and Asp-102 that leads to the formation of an sshb. The formation of this sshb is associated with the $\mathrm{p} K_{\mathrm{a}}$ equalization between the fragments $\mathrm{N} \delta_{1}-\mathrm{O} \delta_{1}$ of His-57 and Asp-102. In synchrony with the sshb formation, the site $\mathrm{N} \epsilon_{2}$ of His-57 changes its $\mathrm{p} K_{\mathrm{a}}$ from 7.4 in the free enzyme to $10-12 \mathrm{p} K_{\mathrm{a}}$ units in the complex. This $\mathrm{p} K_{\mathrm{a}}$ increment permits the abstraction of a proton from Ser-192 $\left(\mathrm{p} K_{\mathrm{a}} \approx 15\right)$ leading to the formation of an alkoxide, which initiates a nucleophilic attack to the peptide bond that is going to be cleaved. Chemical, spectroscopic, and X-ray crystallographic evidence support this mechanism, ${ }^{6-10}$ albeit no consensus about the role that sshb formation may have at the enzymatic sites has been reached. ${ }^{9-13}$

Using a molecular model that mimics the His57-Asp102 interaction, we found that the formation of an sshb induces changes in the electronic properties of the molecular model that can be used to propose an electronic mechanism that gives a cogent rationale

* To whom correspondence should be addressed. Phone: +5626618231 . Fax: +562 6615682. E-mail: ge.mino@uandresbello.edu.

${ }^{\dagger}$ Universidad Andrés Bello.

¥Universidad de Chile.

\section{SCHEME 1: Catalytic Triad of $\alpha$-Chymotrypsine}

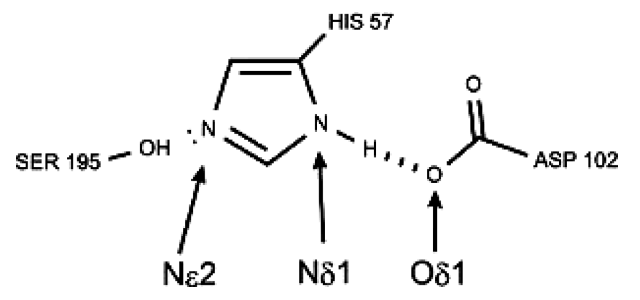

SCHEME 2: Im $\cdots$ Cb Model Used To Simulate the Interaction of His-57 and Asp-102 Residues of a Chymotrypsine

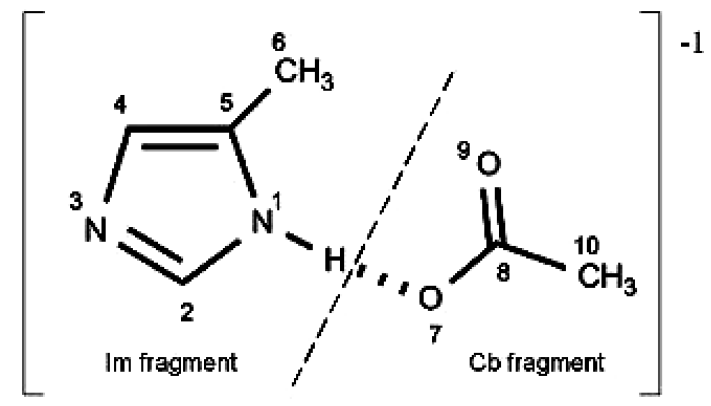

for the role played by these structures on the enzymolysis experimentally observed. The electronic properties used are those defined within the conceptual density functional theory (DFT) of Parr and Yang, ${ }^{14}$ namely, the regional Fukui function and softness. The analysis based on the regional descriptors of DFT is completed by means of the classical molecular electrostatic potential $V(r)$. The purpose of this study is to give qualitative insight into the electronic changes accompanying the formation of short and strong hydrogen bonds and its relationship with the enhanced basicity experimentally observed.

\section{Model and Computational Details}

Our molecular model considers the interaction of 4-methylimidazole (Im) and methyl-carboxilate (Cb) moieties in a similar orientation to that shown by the His- $57 \cdots$ Asp-102 catalytic residues of $\alpha$-chymotrypsine (see Schemes 1 and 2).

The pertinent DFT descriptors of group reactivity are obtained from the condensed to atom nucleophilic Fukui function $f_{k}^{-15}$ 
TABLE 1: Global softness $S\left(R_{\mathrm{N} 1-07}\right)$ and Group Properties $f_{\mathrm{Im}}^{-}\left(R_{\mathrm{N} 1-07}\right), f_{\mathrm{Cb}}^{-}\left(R_{\mathrm{N} 1-07}\right), s_{\mathrm{Im}}^{-}\left(R_{\mathrm{N} 1-07}\right)$, and $s_{\mathrm{Cb}}^{-}\left(R_{\mathrm{N} 1-07}\right)$ Moieties of the Chymotrypsine Model System at Different Stages of the Transition from Normal HB to sshb the Regime for Basis Sets 6-31G(d) and $6-311++\mathbf{G}(\mathbf{d}, \mathbf{p})^{a}$

\begin{tabular}{|c|c|c|c|c|c|c|c|c|c|c|}
\hline \multirow{2}{*}{$\frac{\begin{array}{c}R_{\mathrm{N} 1-\mathrm{O} 7} \\
\text { distance }(\AA)\end{array}}{3.00}$} & \multicolumn{2}{|c|}{$S\left(R_{\mathrm{N} 1-\mathrm{O} 7}\right)\left(\mathrm{eV}^{-1}\right)$} & \multicolumn{2}{|c|}{$f_{\mathrm{Im}}^{-}\left(R_{\mathrm{N} 1-\mathrm{O} 7}\right)$} & \multicolumn{2}{|c|}{$f_{\mathrm{Cb}}^{-}\left(R_{\mathrm{N} 1-\mathrm{O} 7}\right)$} & \multicolumn{2}{|c|}{$s_{\operatorname{Im}}^{-}\left(R_{\mathrm{N} 1-\mathrm{O} 7}\right)\left(\mathrm{eV}^{-1}\right)$} & \multicolumn{2}{|c|}{$s_{\mathrm{Cb}}^{-}\left(R_{\mathrm{N} 1-\mathrm{O} 7}\right)\left(\mathrm{eV}^{-1}\right)$} \\
\hline & 0.159 & 0.213 & 0.019 & 0.007 & 0.981 & 0.993 & 0.003 & 0.002 & 0.156 & 0.211 \\
\hline 2.95 & 0.158 & 0.211 & 0.021 & 0.010 & 0.979 & 0.990 & 0.003 & 0.003 & 0.154 & 0.209 \\
\hline 2.85 & 0.154 & 0.208 & 0.025 & 0.023 & 0.975 & 0.977 & 0.004 & 0.005 & 0.150 & 0.204 \\
\hline 2.80 & 0.152 & 0.207 & 0.029 & 0.043 & 0.971 & 0.957 & 0.004 & 0.009 & 0.147 & 0.198 \\
\hline 2.75 & 0.150 & 0.205 & 0.037 & 0.106 & 0.963 & 0.894 & 0.006 & 0.022 & 0.144 & 0.184 \\
\hline 2.69 & 0.147 & 0.204 & 0.064 & 0.467 & 0.936 & 0.533 & 0.009 & 0.095 & 0.138 & 0.109 \\
\hline 2.68 & 0.147 & 0.204 & 0.078 & 0.569 & 0.922 & 0.431 & 0.012 & 0.116 & 0.135 & 0.088 \\
\hline 2.67 & 0.146 & 0.204 & 0.087 & 0.683 & 0.913 & 0.317 & 0.013 & 0.0140 & 0.134 & 0.065 \\
\hline 2.65 & 0.145 & 0.205 & 0.136 & 0.813 & 0.865 & 0.187 & 0.020 & 0.167 & 0.126 & 0.038 \\
\hline 2.62 & 0.144 & 0.206 & 0.286 & 0.917 & 0.714 & 0.083 & 0.041 & 0.189 & 0.103 & 0.017 \\
\hline 2.61 & 0.144 & 0.206 & 0.449 & 0.933 & 0.551 & 0.067 & 0.065 & 0.192 & 0.079 & 0.014 \\
\hline
\end{tabular}

${ }^{a}$ First entry: B3LYP/6-31G(d) calculation. Second entry: B3LYP/6-311++G(d,p) calculation.

obtained from a single-point scheme by a method described elsewhere ${ }^{16}$ and the corresponding condensed to atom regional softness $s_{k}^{-}$which is obtained from the global softness $S$ as ${ }^{14}$

$$
s_{k}^{-}=S \cdot f_{k}^{-}
$$

Using the normalization condition for $f_{k}^{-}$, namely

$$
\sum_{k} f_{k}=1
$$

we find for a fixed geometry $\Omega$ that

$$
s_{\mathrm{A}}(\Omega)+s_{\mathrm{B}}(\Omega)=S(\Omega)\left[\sum_{k \varepsilon \mathrm{A}} f_{k, \mathrm{~A}}^{-}(\Omega)+\sum_{k \varepsilon \mathrm{B}} f_{k, \mathrm{~B}}^{-}(\Omega)\right]=S(\Omega)
$$

where $s_{\mathrm{A}}^{-}(\Omega)$ and $s_{\mathrm{B}}^{-}(\Omega)$ are the group nucleophilic softnesses associated with fragments $\mathrm{A}$ (representing, for instance, the $\mathrm{Cb}$ moiety) and B (representing, for instance, the Im end of the model H-bonded complex). The Im fragment is composed of the atomic centers $1-6$ including their respective $\mathrm{H}$ atoms and also the $\mathrm{H}$ atom involved in the hydrogen bond, while the $\mathrm{Cb}$ fragment is composed of atomic centers 7-10 including the $\mathrm{H}$ atoms of the methyl group at $\mathrm{C}_{10}$ (see Scheme 2 for atom numbering). Group softnesses in biological systems have proved to be a useful tool to account, for instance, for the orientation of the lumiflavine-1-methylnicotinamide model system during the hydride transfer between the FAD/NADP moieties of the glutathione reductase enzyme. ${ }^{17}$ The group softness associated with fragments $\mathrm{Im}$ and $\mathrm{Cb}$ was evaluated along the compression coordinate defined by the $R\left[\mathrm{~N}_{1}-\mathrm{O}_{7}\right]$ distance, which was varied from 3.0 to $2.5 \AA$, allowing the remaining part of the structure to relax, by a partial optimization procedure performed at the B3LYP/6-31 g(d) and B3LYP/6-311++G(d,p) levels of theory, using the Gaussian03 suite of programs. ${ }^{18}$ The second calculation was performed in order to check whether or not the $s_{\mathrm{Im}}^{-}\left(R_{\mathrm{N} 1-\mathrm{O} 7}\right)$, $s_{C b}^{-}\left(\mathrm{iR}_{\mathrm{N} 1-\mathrm{O} 7}\right)$ profiles are basis set independent. It has been previously reported that the description of strong $\mathrm{H}$-bonded systems requires extended basis sets. ${ }^{19}$

\section{Results and Discussion}

The analysis of the total energy as a function of the $R_{\mathrm{N} 1-\mathrm{O} 7}$ distance of the dyadic model shows a parabolic shape with minima at 2.71 (B3LYP/6-31 g(d)) and $2.70 \AA$ (B3LYP/6$311++\mathrm{G}(\mathrm{d}, \mathrm{p}))$. The proton involved in the $\mathrm{sshb}$ is found at distances from the $\mathrm{N}_{1}$ center that fall within the range 1.08-1.04 $\AA$ for both levels of theory used, when the compression coordinate $R\left[\mathrm{~N}_{1}-\mathrm{O}_{7}\right]$ is varied within the interval 3.0-2.5

The group properties $s_{\mathrm{Im}}^{-}\left(R_{\mathrm{N} 1-\mathrm{O} 7}\right), s_{\mathrm{Cb}}^{-}\left(R_{\mathrm{N} 1-\mathrm{O} 7}\right), f_{\mathrm{Im}}^{-}\left(R_{\mathrm{N} 1-\mathrm{O} 7}\right)$, and $f_{\mathrm{Cb}}^{-}\left(R_{\mathrm{N} 1-\mathrm{O} 7}\right)$ as well as the global softness $S\left(R_{\mathrm{N} 1-\mathrm{O} 7}\right)$ at each stage of the compression process are summarized in Table 1 for both levels of theory used.

The response functions $s_{\mathrm{Im}}^{-}\left(R_{\mathrm{N} 1-\mathrm{O} 7}\right)$ and $s_{\mathrm{Cb}}^{-}\left(R_{\mathrm{N} 1-\mathrm{O} 7}\right)$ plotted along the compression coordinate $R[\mathrm{~N}-\mathrm{O}]$ are depicted in Figure $1 \mathrm{a}$ and $1 \mathrm{~b}$ for both levels of theory. These plots reveal interesting electronic features induced by compression on the HB. First, it may be seen that at the beginning of compression at $R[\mathrm{~N}-\mathrm{O}]=3.0 \AA$ (i.e., at the normal HB stage), the group nucleophilicity of fragment Im is less than that of fragment $\mathrm{Cb}$. This regime is almost maintained until reaching the threshold distance, about 2.65 and $2.75 \AA$ for B3LYP/6-31G(d) and B3LYP/6-311++G(d,p) levels of theory, respectively. Below this distance a sudden nucleophilic deactivation appears, and at the same time, the Im moiety begins to get significantly activated, until a plateau is reached, in both cases, at a distance of $2.5 \AA$, where the denomination of sshb applies.

Note that during the simulated compression process the global softness of the whole system varies smoothly within the narrow ranges of $0.14-0.16$ and $0.20-0.21 \mathrm{eV}^{-1}$ for both the B3LYP/ 6-31G(d) and B3LYP/6-311++G(d,p) calculations, respectively (see Table 1), thereby suggesting that nucleophilic activation of the Im fragment, at the expense of deactivation at the $\mathrm{Cb}$ moiety of the model complex, is mostly governed by changes in the group Fukui function introduced in eq 3 . It seems then that the electronic model that explains the enhanced regional basicity experimentally observed at site $\mathrm{N}_{2}$ of His-57 may be qualitatively accounted for by a nucleophilic activation revealed here at fragment Im containing this site in the model system and described by the group softness $s_{\mathrm{Im}}^{-}\left(R_{\mathrm{N} 1-07}\right)$ index. This result strongly suggest that upon a global external stimulus induced by compression on the whole structure, the system response is to transfer the nucleophilic reactivity from the fragment $\mathrm{Cb}$ toward the fragment $\mathrm{Im}$ in the H-bonded model 

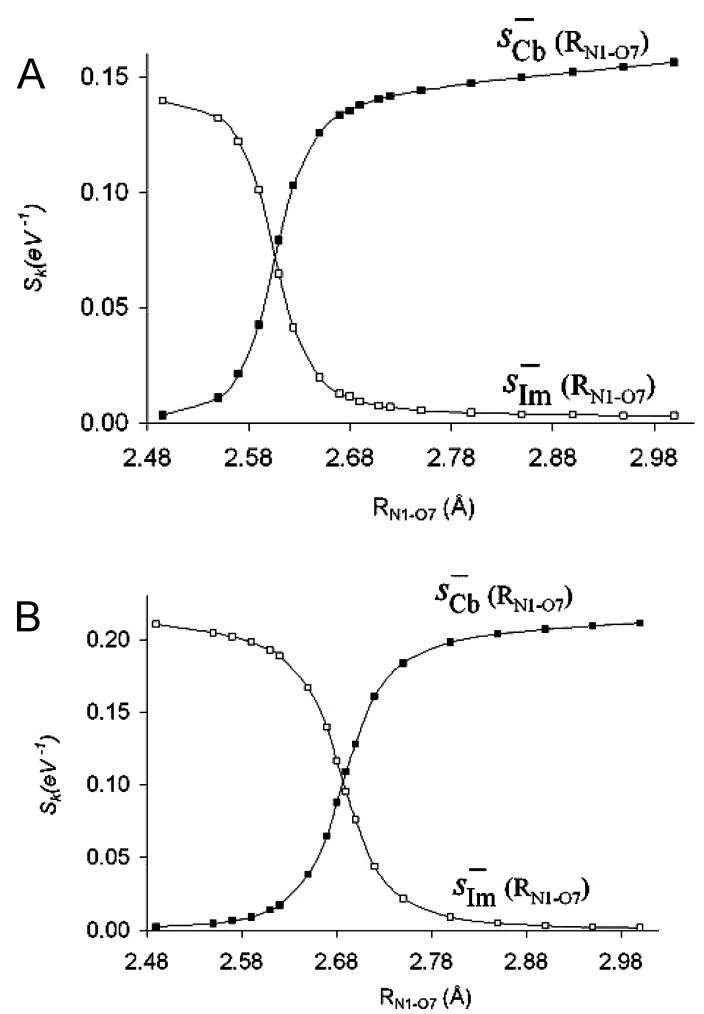

Figure 1. (a) Group softnesses $s_{\mathrm{Cb}}\left(R_{\mathrm{N} 1-\mathrm{O} 7}\right)$ (closed squares) and $s_{\mathrm{Im}}^{-}\left(R_{\mathrm{N} 1-07}\right)$ (open squares) associated with the $\mathrm{Cb}$ and $\mathrm{Im}$ moieties as a function of the compression $R$ [N1-07] coordinate. $\mathrm{B} 3 \mathrm{LYP} / 6-31 \mathrm{G}(\mathrm{d})$ calculation. (b) Group softnesses $s_{\mathrm{Cb}}\left(R_{\mathrm{N} 1-07}\right)$ (closed squares) and $s_{\mathrm{Im}}^{-}\left(R_{\mathrm{N} 1-\mathrm{O} 7}\right)$ (open squares) associated with the $\mathrm{Cb}$ and $\mathrm{Im}$ moieties as a function of the compression $R[\mathrm{~N} 1-\mathrm{O}]$ coordinate. B3LYP/6$311++\mathrm{G}(\mathrm{d}, \mathrm{p})$ calculation.

complex and that this response is mainly driven by changes in the group Fukui function introduced in eq 3.

This electronic mechanism is not new, and it has been proposed to be operative in similar but not equal circumstances. For instance, the change in reactivity observed for the enolate ion from gas to solution phases has been explained on the basis of a second-order static density response of density functional theory. ${ }^{20}$ Therein similar plots to that shown in Figure 1 were reported for the changes in the atomic Fukui functions associated with the two possible nucleophilic centers, namely, the oxygen atom and the $\alpha$ carbon. It was observed that while in the gas phase the preferred site of reaction is the oxygen atom, the reactivity pattern is changed in the aqueous phase as a result of a more favorable preferential solvation at the oxygen atom that causes the nucleophilic reactivity to be transferred to the $\alpha$ carbon in the solution phase. The whole process was shown to be consistently explained within a pair site reactivity model as nonlocal response of the system induced by a local external stimulus. In the present case, however, the external stimulus (i.e., compression) is global since its affects the whole catalytic dyad, but the response of the system is also nonlocal in nature because it involves two different subunits in the H-bonded structure. Despite the fact that the electronic mechanism proposed for the enolate ion and the one proposed here for the chymotrypsine active site differs mainly on the kind of environmental effects considered, solvation in the former and compression in the later, they share a common nonlocal response of the system, and both processes may be consistently explained on the basis of the static Fukui response function proposed earlier. $^{20}$

In order to reinforce the results discussed above, a further study was performed to check whether or not the nucleophilic reactivity transfer from the $\mathrm{Cb}$ toward the Im fragments was consistent with the enhanced basicity experimentally observed at the site $\mathrm{N} \epsilon_{2}$ of His-57. The study considers a local analysis performed on the basis of the molecular electrostatic potential $V(\mathbf{r})$. Figures 2 and 3 show a qualitative representation of the changes in $V(\mathbf{r})$ projected upon an electron density isosurface of 0.0004 and $0.0020 \mathrm{e} / \mathrm{b}^{3}$, respectively, for the selected distances $R\left[\mathrm{~N}_{1}-\mathrm{O}_{7}\right] 3.00,2.70$, and $2.50 \AA$. Upon compression, a change toward more negative values of $V(\mathbf{r})$ is obtained at the Im ring, the more negative value being located at the site of biochemical interest, namely, $\mathrm{N}_{3}$ in Scheme 2.

Following a suggestion by a reviewer, an alternative explanation for the increase in basicity at the imidazole moiety of the model H-bonded complex involves the decrease of the barrier of proton transfer when the distance is shortened and some degree of proton transfer takes place. In order to discuss this issue, proton-transfer profiles obtained from linear transit calculations at the B3LYP/6-311G $++(d, p)$ level of theory were performed. As seen in Figure 4, shortening of the donor-acceptor $\mathrm{H}$-bond distance results in a decrease of the barrier to proton transfer, thereby showing that partial proton transfer may be one of the factors contributing to the enhanced basicity experimentally observed. This effect however does not contradict the electronic mechanism based on the pair site reactivity model proposed above.

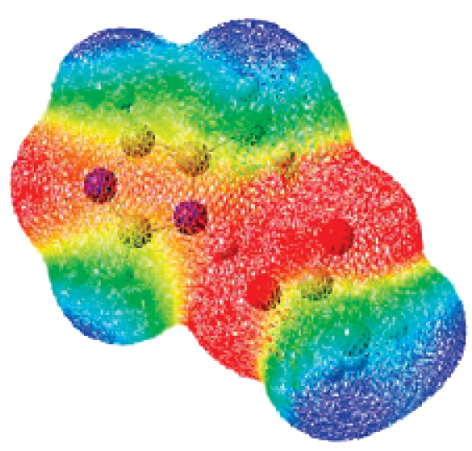

(a)

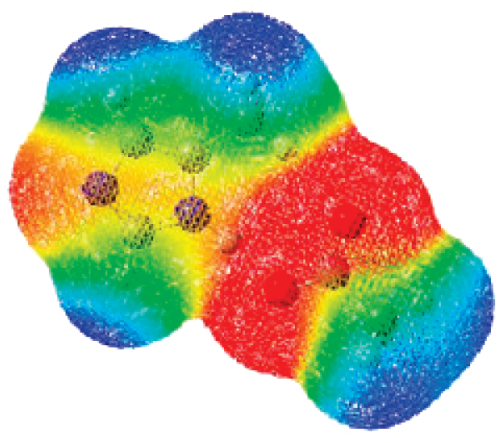

(b)

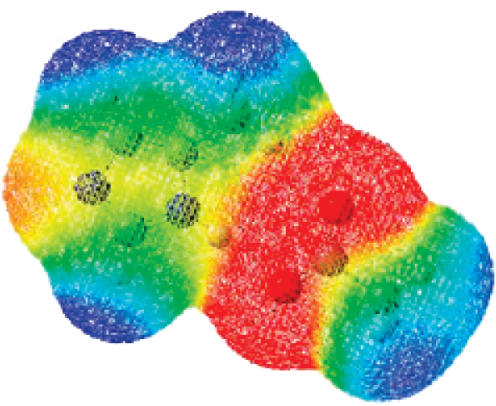

(c)

Figure 2. Projection of the molecular electrostatic potential $V(\mathbf{r})$ upon an electronic isodensity surface of 0.0004 e/ $\mathrm{b}^{3}$ at (a) 2.50 , (b) 2.70 , and (c) 2.95 A. [color scale: -102 (red), -82 (green), and -61 (blue) kcal/mol]. B3LYP/6-31G(d) calculation. 


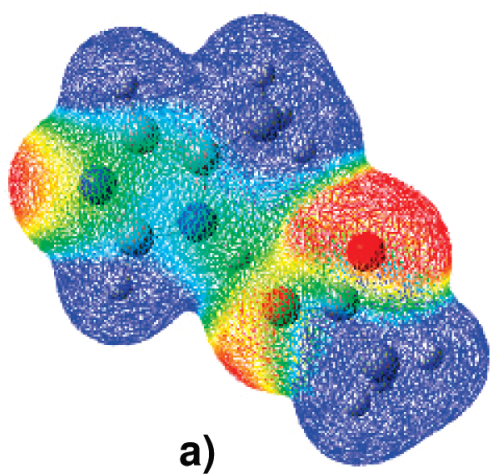

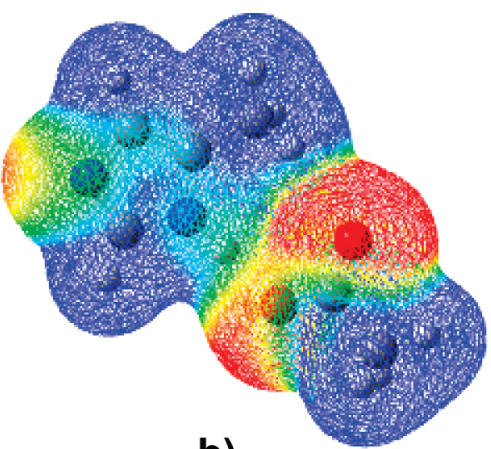

b)

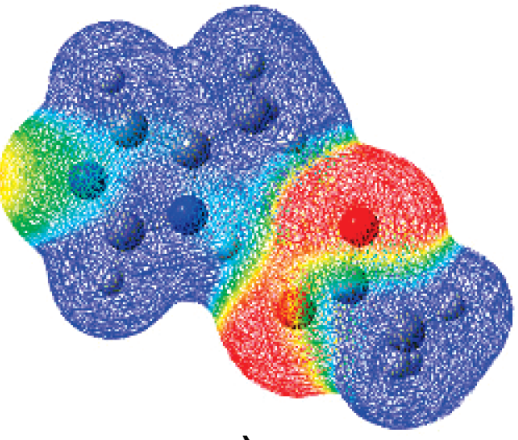

c)

Figure 3. Projection of the molecular electrostatic potential $V(r)$ upon an electronic isodensity surface of 0.0020 e/ $\mathrm{b}^{3}$ at (a) 2.50 , (b) 2.70 , and (c) 3.00 Å. [color scale: -119 (red), -104 (green), and -88 (blue) kcal/mol]. B3LYP/6-311G++(d,p) calculation.

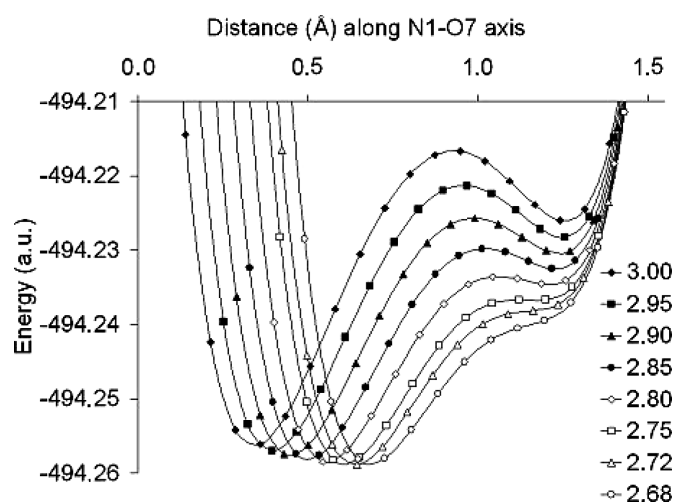

Figure 4. Proton-transfer profiles from linear transit calculations at the B3LYP/6-311++G(d,p) level of theory for selected $R_{\mathrm{N} 1-\mathrm{O} 7}$ distances.

\section{Concluding Remarks}

The role played by the short and strong hydrogen bond on the mechanism of action of a model chymotrypsine active has been discussed. The results reveal that at short HB distances, where the denomination of sshb applies, the nucleophilic reactivity is transferred from the carboxylate moiety toward the imidazole fragment, as a result of a global compression on the whole dyadic structure, the regional nucleophilicity being represented in the present model by the group softnesses at the $\mathrm{Cb}$ and $\mathrm{Im}$ end of the H-bonded model complex. The leading term of group softness at each fragment is the group Fukui function. This result strongly suggests that the nucleophilic activation/deactivation patterns observed at sshb distance of the $\mathrm{Im}$ and $\mathrm{Cb}$ moieties, respectively, may be explained on the basis of a pair site reactivity model previously derived from the second-order static density response function (static Fukui response function) that probes here an unusual "medium effect" in the form of compression of the system by an external agent, represented in the experimental model of catalysis proposed by Frey, by a steric strain produced by the biological environment on the catalytic triad.

Acknowledgment. This work was supported by Fondecyt, grant no. 1070715. G.M. thanks the Universidad Andrés Bello and Comisión Nacional de Ciencia y Tecnología for a doctoral fellowship awarded to him.

\section{References and Notes}

(1) Hibbert, F.; Emsley, J. Adv. Phys. Org. Chem. 1990, 26, 255.

(2) Cleland, W. W. Biochemistry 1992, 31, 317.

(3) Kuhn, P.; Knapp, M.; Soltis, S. M.; Ganshaw, G.; Thoene, M.; Bott, R. Biochemistry 1998, 37, 13446.

(4) Massiah, M. A.; Viragh, C.; Reddy, P. M.; Kovach, I. M.; Johnson, J.; Rosenberry, T. L.; Mildvan, A. S. Biochemistry 2001, 40, 5682.

(5) Stranzl, G. R.; Gruber, K.; Steinkellner, G.; Zangger, K.; Schwab, H.; Kratky, C. J. Biol. Chem. 2004, 279, 3699.

(6) Cassidy, C.; Lin, J.; Frey, P. A. Biochemistry 1997, 36, 4576.

(7) Tobin, J. B.; Whitt, S. A.; Cassidy, C. S.; Frey, P. A. Biochemistry 1995, 34, 6919.

(8) Cloninger, M. J.; Frey, P. A. Bioorg. Chem. 1998, 26, 323.

(9) Frey, P. A.; Whitt, S. A.; Tobin, J. B. Science 1994, 264, 1927.

(10) Cleland, A.; Kreevoy, M. Science 1994, 264, 1887.

(11) Warshel, A.; Papazyan, A.; Kollman, P. Science 1995, 269, 102.

(12) Warshel, A.; Papazyan, A. Proc. Natl. Acad. Sci. 1996, 93, 13665.

(13) Fuhrmann, C. N.; Daugherty, M. D.; Agard, D. A. J. Am. Chem. Soc. 2006, 128, 9086

(14) Parr, R. G.; Yang, W. Density Functional Theory of Atoms and Molecules; Oxford: New York, 1989.

(15) Yang, W.; Mortier, W. J. J. Am. Chem. Soc. 1986, 108, 5708.

(16) Contreras, R. R.; Fuentealba, P.; Galvan, M.; Perez, P. Chem. Phys. Lett. 1999 304, 405.

(17) Rivas, P.; Zapata-Torres, G.; Melin, J.; Contreras, R. Tetrahedron 2004, 60, 4189.

(18) Frisch, M. J., et al. Gaussian 03, Revision D.01; Gaussian, Inc.: Pittsburgh, PA, 2003.

(19) Filarowski, A.; Majerz, I. J. Phys. Chem. A 2008, 112, 3119.

(20) Contreras, R.; Domingo, L. R.; Andŕes, J.; Ṕerez, P.; Tapia, O. J. Phys. Chem. A 1999, 103, 1367.

JP902756X 